\title{
Herbal immunomodulators as add on treatment in asymptomatic and mildly symptomatic COVID-19 confirmed cases: findings from a prospective single centre clinical trial
}

\author{
M. Ravi Kumar Reddy, K. R. Hari Venkatesh*, J. Jothilakshmi
}

Research and Development-Healthcare, Sriveda Sattva Private Limited (Sri Sri Tattva), Bengaluru, Karnataka, India

Received: 31 March 2021

Revised: 30 April 2021

Accepted: 01 May 2021

\section{*Correspondence:}

Dr. Hari Venkatesh K. R.,

Email: drharivenkatesh@srisritattva.com

Copyright: (c) the author(s), publisher and licensee Medip Academy. This is an open-access article distributed under the terms of the Creative Commons Attribution Non-Commercial License, which permits unrestricted non-commercial use, distribution, and reproduction in any medium, provided the original work is properly cited.

\begin{abstract}
Background: The aim of the present study was to evaluate safety and efficacy of Sri Sri Tattva ${ }^{\mathrm{TM}}$ immunity products, a combination of single herb and poly herb formulations in COVID-19 patients by assessing their clinical features and immune markers. The products when administered orally for a period of 14 days in COVID-19 patients demonstrated significant improvement in clinical symptoms.

Methods: Patients with fever, cough, sore throat started responding to the treatment from day 3 and most of the patients were clinically free of symptoms by day 7. Early recovery from signs and symptoms was observed in most of the patients. Sri Sri Tattva ${ }^{\mathrm{TM}}$ immunity products has also demonstrated an excellent clinical improvement and immune markers including tumor necrosis factor (TNF-alfa), interleukin (IL-6), interferon (IFN-beta), D-dimer, ferritin, lactate dehydrogenease (LDH) and C-reactive protein (CRP) in COVID-19 patients.

Results: The final results of this study showed that Sri Sri Tattva ${ }^{\mathrm{TM}}$ immunity products reduced the time to clinical cure and number of days in achieving negative real time polymerase chain reaction (RT-PCR) by early viral clearance when given along with standard of care thus reduced the duration of hospitalization.

Conclusions: In the present study, $82 \%$ of COVID-19 patients cleared the virus within 10-14 days of study period reducing the duration of hospitalization. None of the patients progressed to severe COVID-19 and succumbed to the disease. This clearly indicates that Sri Sri Tattva ${ }^{\mathrm{TM}}$ immunity products when administered orally along with standard of care has definitive role in the management of COVID-19 patients.
\end{abstract}

Keywords: COVID-19, Immunity, RT-PCR, Sri Sri Tattva ${ }^{\mathrm{TM}}$, Herbal immunomodulators

\section{INTRODUCTION}

The new coronavirus (COVID-19) has posed a situation that warrants immediate global attention. Despite availability of many antiviral drugs in the management of COVID-19 symptoms, there are no specific preventive medicines thus far available in the market. Even though very few effective antivirals for other indications are in the market, they do not meet the production demands during the pandemic situation, to cater to the needs of the masses worldwide. Furthermore, vast majority of drugs of this category are highly responsible for development of drug resistance. In addition, emergence of mutant strains of the virus, more virulent strain, exorbitant costs of available drugs, time lag between vaccine developments and mass casualties gave rise to difficult problems. On the other side, 
complementary and alternative medicine (CAM) delivers an ample of exciting preventive options in patients. Many a times, herbs demonstrate an array of therapeutic activities and can be successfully tapped for controlling pandemic flu per se. CAM has been used for the welfare of the humankind, especially when typical modern medicine has failed to meet the expectations. Time and again, CAM has been used in concurrence with conventional medicine to obtain synergistic effects in the recent past. ${ }^{1-3}$

The traditional system of medicine has been popularly used since many years now and a number of medicinal plants of the Indian subcontinent have been taken for advantage in addressing almost every human ailment. Several antiviral agents were isolated from plants as a result of chemical and pharmacological studies in the recent years and many have been derived from leads based on Ayurveda and other traditional medicine principles. In last few years, there is a significant upsurge in the therapeutic options for viral infections, however, there is an emergence of resistant viruses as well. The subsequent process of getting rid of resistant strains is a strong cause for their therapeutic failure. ${ }^{4,5}$ Furthermore, many of the licensed drugs are toxic as well as being expensive, thus the search for potential sources for the development of new drugs or supplements is very important. Based on ayurveda and siddha traditional herbal medicine, several antiviral studies were performed to detect active natural products in higher plants. Ayurveda sees all life and nature as constantly evolving towards a higher level of consciousness. ${ }^{6}$ Ayurvedic formulations have an impact at this higher level of consciousness, as well as the more gross body level. Asian ethnicity connects to ayurveda than the western world due to multiple reasons. The traditional knowledge, with a keen understanding of the universal laws and practical observations give them the indication about the holistic approach of Ayurveda and its potential in alleviating many health-related problems afflicting the whole of humanity. Herbal medicines and purified natural products provide a rich resource for novel antiviral drug development. ${ }^{7,8}$ Sri Sri Tattva ${ }^{\mathrm{TM}}$ immunity products are a combination of single herb and poly herb formulations which are potent antivirals, antibacterial and anti-inflammatory along with immune boosting ingredients. Amruth, turmeric plus, tulasi arka and shakti drops (polyherbal combination) have been suggested in the treatment of viral fevers. Amruth, is a plant with as diverse a role as Tinospora cordifolia is a multifaceted resource for all forms of life. There are reports that the plant extracts have active compounds in the form of alkaloids, glycosides, lactones and steroids. All these bio-active compounds have immunomodulatory and physiological roles of different types, thereby demonstrating the diverse versatility of the plant. Turmeric, a curcuminoid-piperine combination also significantly improves oxidative and inflammatory status. Curcuminoids could therefore be regarded as natural, safe and effective CRP lowering agents. Ocimum, also known as holy basil, is indigenous to the Indian continent and highly revered for its medicinal uses within the ayurveda. Many in-vitro, animal and human studies provide evidence to tulasi having multiple therapeutic actions including adaptogenic, antimicrobial, anti-inflammatory, cardioprotective and immunemodulatory effects. Shakti drops an ayurvedic polyherbal formulation is an effective medicine for immunemodulatory activity. RT-PCR negativity of the throat/nasal swab is an important indicator of elimination of virus particles from the body and active immune status against corona virus in COVID-19. In many studies COVID-19 patients showed that the mean contagious period of SARSCoV-2 infected patients was around 20 days. ${ }^{9}$ This clinical trial was conducted to evaluate the safety and efficacy of Sri Sri Tattva ${ }^{\mathrm{TM}}$ immunity product as an add-on treatment in asymptomatic and mildly symptomatic COVID-19 confirmed cases.

\section{METHODS}

The trial was conducted in Bangalore medical college and research institute, Bengaluru, Karnataka, India with Jayanthi CR as principal investigator, post the institutional ethics committee approval/favorable opinion on the trial proposal. The trial was prospectively registered in the indian clinical trial registry, CTRI/2020/06/025592. The objective of this study was to evaluate the safety \& efficacy of herbal immunomodulators (Sri Sri Tattva ${ }^{\mathrm{TM}}$ immunity products) as add on treatment in asymptomatic and mildly symptomatic COVID-19 confirmed cases. The primary outcome was to detect early recovery in the signs and symptoms of cases of mild symptomatic COVID-19 patients and asymptomatic cases by adding ayurvedic medicines (Sri Sri Tattva ${ }^{\mathrm{TM}}$ ) which are effective in enhancing immunity of body and reducing the symptoms such as fever, cough and sore throat from day 1 and day 14 and the secondary outcomes were to assess the improvement in laboratory parameters. The trial was initiated on 21 May 2020 and the first subject was enrolled on 4 June 2020 . There were a total of 12 screen failures and the study duration was from 4 June 2020 to 26 June 2020. All the 40 enrolled subjects were administered study drug along with standard of care (SoC), daily after food for 14 consecutive days. Medical history and demographic data including sex, age, body weight $(\mathrm{kg})$ and height $(\mathrm{cm})$ and habits were recorded during general screening of volunteers. Each subject underwent a complete general physical examination. All inclusion and exclusion criteria's were reviewed for each subject meticulously, prior to his/her enrollment into this study. Post confirmation of COVID-19 through RT-PCR test on day 1 , the presented regime was administered to the enrolled patients of shakti drops (dosage $=5$ drops, 3 times a day), amruth tablet (dosage $=1$ tablet, 2 times a day), turmeric plus tablet (dosage $=1$ tablet, 2 times a day), tulasi arka (dosage $=10$ drops, 3 times a day). The formulation was prepared in the laboratory with authenticated organic plant drugs. Any other herbal supplements, ayurveda, homeopathic or OTC products intended to treat present illness apart from study drug with SoC were not allowed/prohibited during the study period. Subjects were 
prohibited from smoking during their participation in the study (starting from the screening to the end of study). Clinical signs and symptoms, TNF-alfa, IL-6, IFNlambda, IFN-beta, D-dimer, ferritin, LDH and CRP were monitored for improvement on day 0 and day 14 Complete blood count (CBC), renal function test (RFT) and liver functions test (LFT) were monitored on day 0 and day 14 for safety assessment. Along with the laboratory parameters patients were also monitored for vitals, physical examination and adverse events for safety (Table 1). In accordance with the government of India revised discharge policy for covid hospitalized patients dated 26 May 2020, RT-PCR test was performed on day 1 and day 10 in mildly symptomatic patients. If the patient was asymptomatic then RT-PCR was performed on day 7. Student t-test was employed for analyzing all the biomarker values with baseline data as co-variate, while $p$ value $<0.05$ was considered as statistical significance for the study. The inclusion criteria was age limit 18 to 60 years, gender both male and female, confirmed asymptomatic and mildly symptomatic cases of COVID19 infection, uncomplicated cases of COVID-19 patients on allopathic medication, patients in whom ventilator support was not required, patients with no associated co morbidities, patients willing to give informed consent to participate in the clinical trial. While the exclusion criteria was COVID-19 positive patients above 60 years of age and below 18 years with symptoms, patients with associated comorbidities like hypertension, type 2 or type 1 diabetes mellitus, chronic or acute renal failure, patients on immuno-suppression therapy, pregnant women or lactating mothers patients in advanced stage of disease requiring emergency medical intervention like pneumonia, bronchial asthma, organ failure.

Table 1: Schedule of assessments.

\begin{tabular}{|c|c|c|c|c|c|}
\hline $\begin{array}{l}\text { S. } \\
\text { no. }\end{array}$ & Procedures & $\begin{array}{l}\text { Screening } \\
\text { (day 0) }\end{array}$ & $\begin{array}{l}\text { Visit } 1 \\
\text { (day 1) }\end{array}$ & $\begin{array}{l}\text { Visit } 2 \text { (day } \\
\text { 14) }\end{array}$ & $\begin{array}{l}\text { Visit } 3 \\
\text { (day 30) } \\
\text { telephonic }\end{array}$ \\
\hline 1 & Inclusion and exclusion criteria & $\mathrm{X}$ & $\mathrm{X}$ & - & - \\
\hline 2 & Informed consent & $\mathrm{X}$ & - & - & - \\
\hline 3 & Demographics & $\mathrm{X}$ & - & - & - \\
\hline 4 & Medical and medication history & $\mathrm{X}$ & - & - & - \\
\hline 5 & Vital signs and physical examination & $\mathrm{X}$ & $\mathrm{X}$ & $\mathrm{X}$ & - \\
\hline 6 & $\begin{array}{l}\text { Laboratory investigations (TNF-alfa, IL-6, IFN- } \\
\text { lambda, IFN-beta, D-dimer, ferritin, LDH, CRP, } \\
\text { CBC, LFT, RFT) }\end{array}$ & $\mathrm{X}$ & - & $\mathrm{X}$ & - \\
\hline 7 & Co-morbid conditions & $\mathrm{X}$ & - & - & - \\
\hline 8 & Concomitant medications & $\mathrm{X}$ & $\mathrm{X}$ & $\mathrm{X}$ & - \\
\hline 9 & IP dispensing & - & $\mathrm{X}$ & - & - \\
\hline 10 & Adverse events & - & - & $\mathrm{X}$ & $X$ \\
\hline
\end{tabular}

\section{RESULTS}

Total 40 subjects participated in this study of which 19 subjects were male $(46 \%)$ and 21 subjects were female $(54 \%)$. Mean age of participants was 39 years. Table 2 reflects demographics of the study subjects. Figure 1 gives an outline of total number of subjects screened, number of screen failures, enrolled, completed the study. In the present study, 31 patients $(81.5 \%)$ cleared the virus within 10-14 days. None of the patients progressed to severe COVID-19 and none of the patients succumbed to the disease. Vitals like temperature, systolic and diastolic blood pressure, pulse rate, heart rate and respiratory rate measured and recorded at all the visits. There was no clinically significant abnormality observed both the groups inferring the active product is safe for administration. However, there were 4 adverse events (2 nausea, 1 headache, 1 stomach upset) observed for 4 different subjects which were categorized as mild to moderate in severity with none of the events were judged to be related to study product in the investigator's opinion. Subjects were followed up telephonically on day 30 , for evaluating their overall well-being and safety/adverse events. None of the enrolled subjects had serious adverse events requiring prolonged hospitalization or death. The safety laboratory parameters RFT and LFT were within normal limits at screening and on day 14.

The values decreased by day 14 and reached statistical significance for IL-6, TNF-alpha and IFN-beta. No improvement observed in IFN-lambda values between the two visits (day 0 and day 14).

In the present study, in mild symptomatic cases IL-6 is 23.2 $\mathrm{Pg} / \mathrm{ml}$ on day 0 and $21.8 \mathrm{Pg} / \mathrm{ml}$ on day 14 . Reduction in IL6 in mild symptomatic patients is statistically significant $(\mathrm{p}=0.0056)$. Mean IL-6 in asymptomatic patients is 22.3 $\mathrm{Pg} / \mathrm{ml}$ on day 0 and $21.1 \mathrm{Pg} / \mathrm{ml}$ on day 14 . Reduction in IL6 in asymptomatic patients is statistically significant $(\mathrm{p}=0.0035)$.

In the present study mean TNF alfa in mild symptomatic patients is $13.6 \mathrm{Pg} / \mathrm{ml}$ on day 0 and $12.4 \mathrm{Pg} / \mathrm{ml}$ on day 14 which is statistically highly significant $(\mathrm{p}=0.0002)$. In 
asymptomatic cases TNF-alfa is $12.9 \mathrm{Pg} / \mathrm{ml}$ on day 0 and $12.0 \mathrm{Pg} / \mathrm{ml}$ on day 14 which is statistically significant $(\mathrm{p}=0.0143)$.

In the present study mean IFN-beta in mild symptomatic patients is $17.5 \mathrm{Pg} / \mathrm{ml}$ on day 0 and it increased to 17.7 $\mathrm{Pg} / \mathrm{ml}$ at day 14 which is statistically significant $(\mathrm{p}=0.0183)$. In asymptomatic cases IFN-beta is $17.0 \mathrm{Pg} / \mathrm{ml}$ on day 0 and $17.3 \mathrm{Pg} / \mathrm{ml}$ at day 14 which is statistically significant $(\mathrm{p}=0.0019)$.

In the present study mean D-dimer is showing decreasing trend from day 0 to day 14 in mild symptomatic patients. In asymptomatic patients D-dimer is $0.78 \mathrm{mcg} / \mathrm{ml}$ on day 0 and $0.59 \mathrm{mcg} / \mathrm{ml}$ on day 14 . D-dimer decreased significantly in asymptomatic group from day 0 to day 14 $(\mathrm{p}=0.0013)$.

In the present study mean ferritin value in mild symptomatic patients on day 0 is $231.53 \mu \mathrm{g} / 1$ and 107.08 $\mu \mathrm{g} / \mathrm{l}$ on day 14 . Reduction in ferritin values in mild symptomatic group is statistically significant $(\mathrm{p}=0.0285)$.
In the present study mean LDH values on day 0 in mild symptomatic patients is $307.58 \mathrm{U} / 1$ and 213.21 on day 14 . The reduction in LDH value in mild symptomatic patients is statistically significant $(\mathrm{p}=0.0366)$. In asymptomatic patients mean LDH values on day 0 is $237.79 \mathrm{U} / 1$ and 194.26 U/1 on day 14. The reduction in LDH values in asymptomatic group is statistically highly significant $(\mathrm{p}<0.0001)$.

In the present study mean CRP values in mild symptomatic patients on day 0 is $13.73 \mathrm{mg} / \mathrm{l}$ and $3.54 \mathrm{mg} / \mathrm{l}$ on day 14 . The reduction in CRP values in mild symptomatic group is statistically significant $(\mathrm{p}=0.0444)$. In asymptomatic group CRP value is $2.69 \mathrm{mg} / \mathrm{l}$ on day 0 and $0.85 \mathrm{mg} / \mathrm{l}$ on day 14 . Decrease in CRP in asymptomatic patients is also statistically significant $(\mathrm{p}=0.0424)$.

The values decreased by day 14 and reached statistical significance across all these 4 markers in asymptomatic and mild symptomatic groups, except for D-dimer in mild symptomatic patients and ferritin in asymptomatic patients which did not reach a significance.

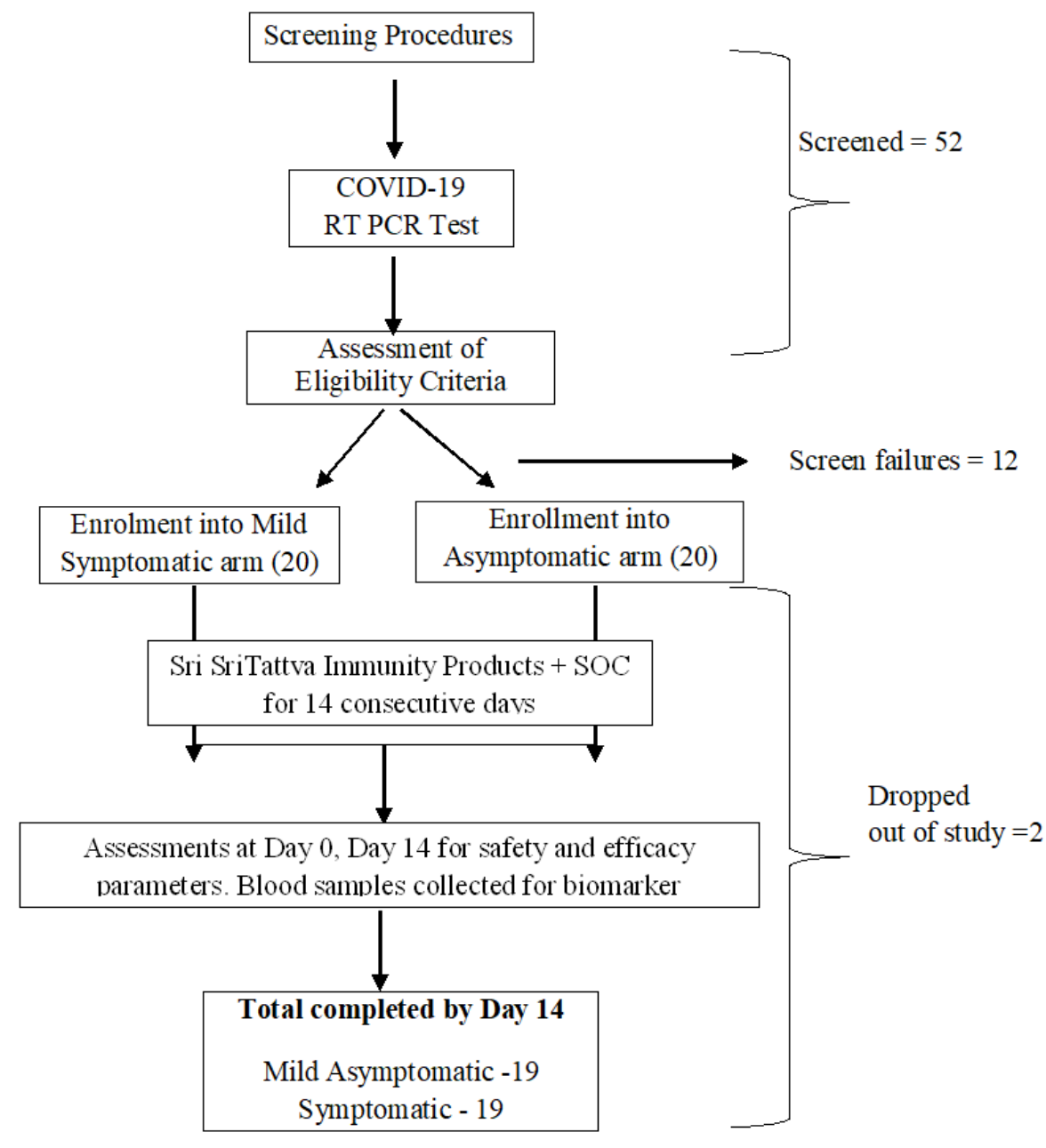

Figure 1: Patients screened, enrolled and completed the study. 
Table 2: Subject demographics.

\begin{tabular}{|llll|}
\hline S. no. & Parameters & Male & Female \\
\hline $\mathbf{1}$ & Age (in years) & 41 & 37 \\
\hline $\mathbf{2}$ & Height (in cms) & 165 & 157 \\
\hline $\mathbf{3}$ & Weight (in $\mathrm{kgs})$ & 72.2 & 69.4 \\
\hline $\mathbf{4}$ & BMI (in $\mathrm{kg} / \mathrm{m}^{2}$ ) & 26.51 & 28.16 \\
\hline
\end{tabular}

\section{DISCUSSION}

Recently, liver function was identified as an important predictor for COVID-19 patient mortality and the study findings suggested that SARS-CoV-2 may directly bind to ACE2-positive cholangiocytes and therefore, liver abnormalities in COVID-19 patients may be due to cholangiocyte dysfunction and other causes, such as drug induced and systemic inflammatory response-induced liver injuries. ${ }^{10,11}$ Regarding the specific and dynamic pattern of liver injury parameters, Lei et al in a wide retrospective multicenter study involving a COVID-19 cohort-derived data set of 5771 patients, concluded that aspartate transaminase is strongly associated with mortality risk compared to other parameters, reflecting liver injury. ${ }^{12}$ The safety laboratory parameters RFT and LFT were within normal limits at screening and on day 14. Therefore, it can be concluded that the ingredients of Sri Sri Tattva ${ }^{\mathrm{TM}}$ immunity products formulations are completely safe for human oral consumption.

In patients with COVID-19, there is upregulation of proinflammatory cytokines in the blood (Figure 2), including $\mathrm{TNF}$ and patients in intensive care units have increased concentrations of many cytokines. In the present study mean TNF-alfa in mild symptomatic and asymptomatic cases decreased by day 14 and reached is statistical significance. Previous retrospective studies indicated that an elevated level of interleukin-6 (IL-6) was associated with a high case fatality of COVID-19 infection. In this study, the IL-6 values reduced significantly in mild symptomatic cases and in mild symptomatic patients. The main function of IFN-lambda is to prevent viral infection by establishing an antiviral state and, if infected, to slow viral replication and dissemination. In present study there is no improvement in IFN-lambda values from day 0 to day 14. However longer studies may be required to evaluate this parameter. IFN- $\beta$ la is an important prognostic marker in COVID-19 and potently inhibits SARS coronavirus replication. In the present study mean IFN-beta in mild symptomatic patients is $17.5 \mathrm{Pg} / \mathrm{ml}$ on day 0 and it increased to $17.7 \mathrm{Pg} / \mathrm{ml}$ at day 14 which is statistically significant $(\mathrm{p}=0.0183)$. In asymptomatic cases IFN-beta is 17.0 $\mathrm{Pg} / \mathrm{ml}$ on day 0 and $17.3 \mathrm{Pg} / \mathrm{ml}$ at day 14 which is statistically significant $(\mathrm{p}=0.0019)$.

D-dimer is a marker of disseminated intravascular coagulation (DIC) and associated with worst prognosis. Tang et al also recently highlighted that the vast majority of COVID-19 patients who died during hospital stay fulfilled the criteria for diagnosing disseminated intravascular coagulation (71.6 versus $0.6 \%$ in survivors). In the present study mean D-dimer is demonstrated decreasing trend from day 0 to day 14 in mild symptomatic and asymptomatic patients by day 14 . Ferritin are generally within the normal range in patients with non-severe disease (according to the national health commission of China guidelines for COVID-19 severity classification). However, hyperferritinemia (ferritin level $>400 \mu \mathrm{g} / \mathrm{l}$ ), was observed in patients with severe disease. In the present study mean ferritin value in mild symptomatic patients and asymptomatic group reached significance by day 14 . Severe infections may cause cytokine-mediated tissue damage and LDH release. Since LDH is present in lung tissue (isozyme 3), patients with severe COVID-19 infections can be expected to release greater amounts of $\mathrm{LDH}$ in the circulation, as a severe form of interstitial pneumonia, often evolving into acute respiratory distress syndrome, is the hallmark of the disease. It was also one of the biomarkers most strongly associated with ARDS mortality. ${ }^{13,14}$ Hence LDH is an important laboratory parameter in assessing the severity of tissue injury. In the present study mean LDH values on day 0 reduced by day 14 in mild symptomatic and also in asymptomatic patients, reached statistically significant. Importantly, CRP has been associated with disease development and is an early predictor for severe COVID-19. ${ }^{15}$ The increased CRP levels were likely due to COVID-19 related acute inflammatory pathogenesis during which multiple cytokines were released and their amount was associated with disease severity. ${ }^{16}$ Hence CRP is an important lab parameter in assessing the severity of inflammation. In the present study mean CRP values in mild symptomatic patients on day 0 is $13.73 \mathrm{mg} / \mathrm{l}$ and $3.54 \mathrm{mg} / \mathrm{l}$ on day 14 . The reduction in CRP values in mild symptomatic group is statistically significant ( $\mathrm{p}=0.0444)$. In asymptomatic group CRP value is $2.69 \mathrm{mg} / \mathrm{l}$ on day 0 and $0.85 \mathrm{mg} / \mathrm{l}$ on day 14 . Decrease in CRP in asymptomatic patients is also statistically significant $(\mathrm{p}=0.0424)$. Figure 3 depicts changes in biomarkers on day 0 and day 14 .

In present study patients with clinical symptoms including fever, cough, sore throat and respiratory distress were assessed from baseline and day 14. There was clinically significant improvement in symptoms in the patients during the course of the study. Early recovery from signs and symptoms was observed in most of the patients. None of the patients progressed to pneumonia or respiratory failure. Asymptomatic patients were stable throughout the study period and none of them progressed to active disease till the end of the study. 

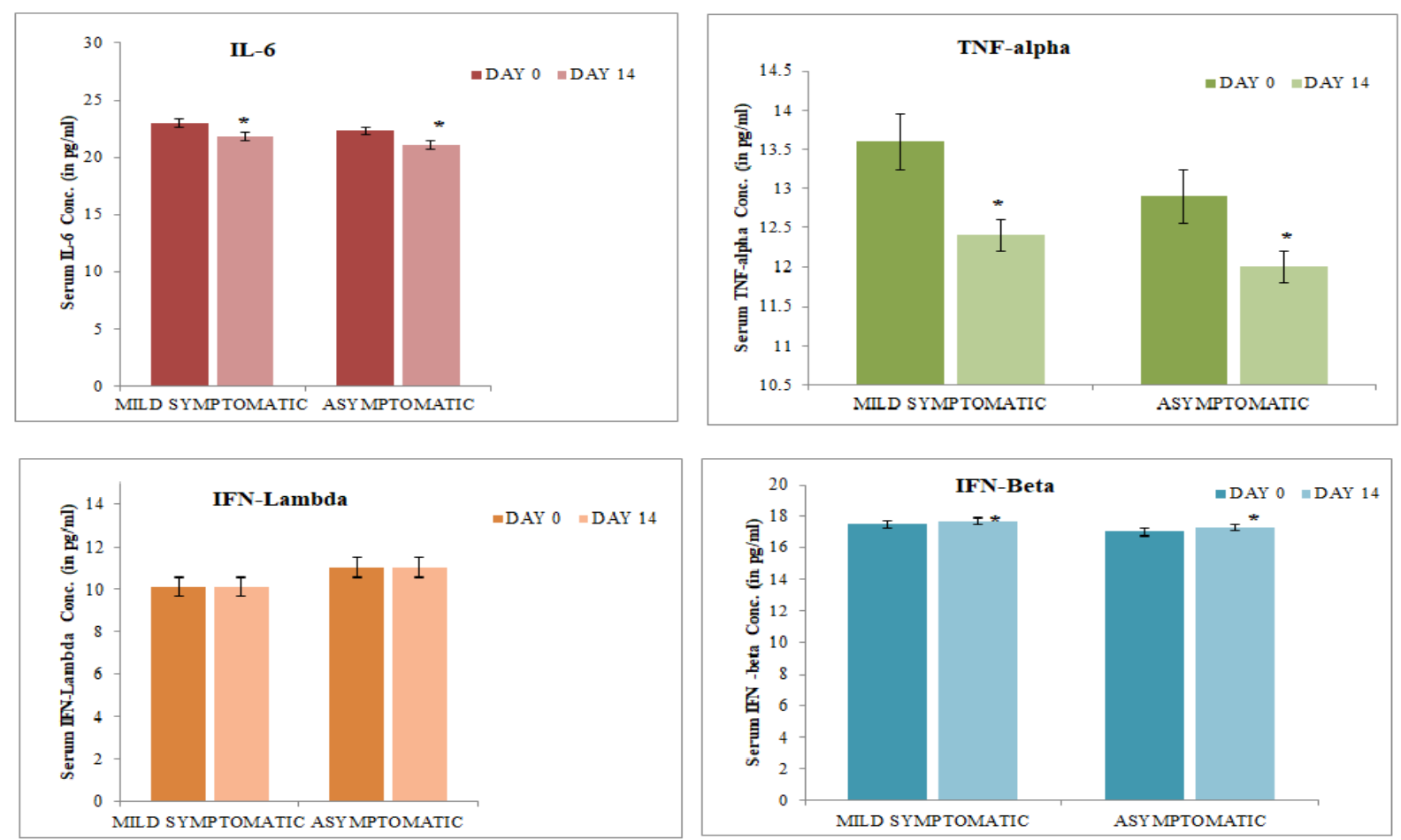

Figure 2: IL-6, TNF-alpha, IFN-lambda and beta values were compared for mild symptomatic and asymptomatic arms on day 0 and day 14.
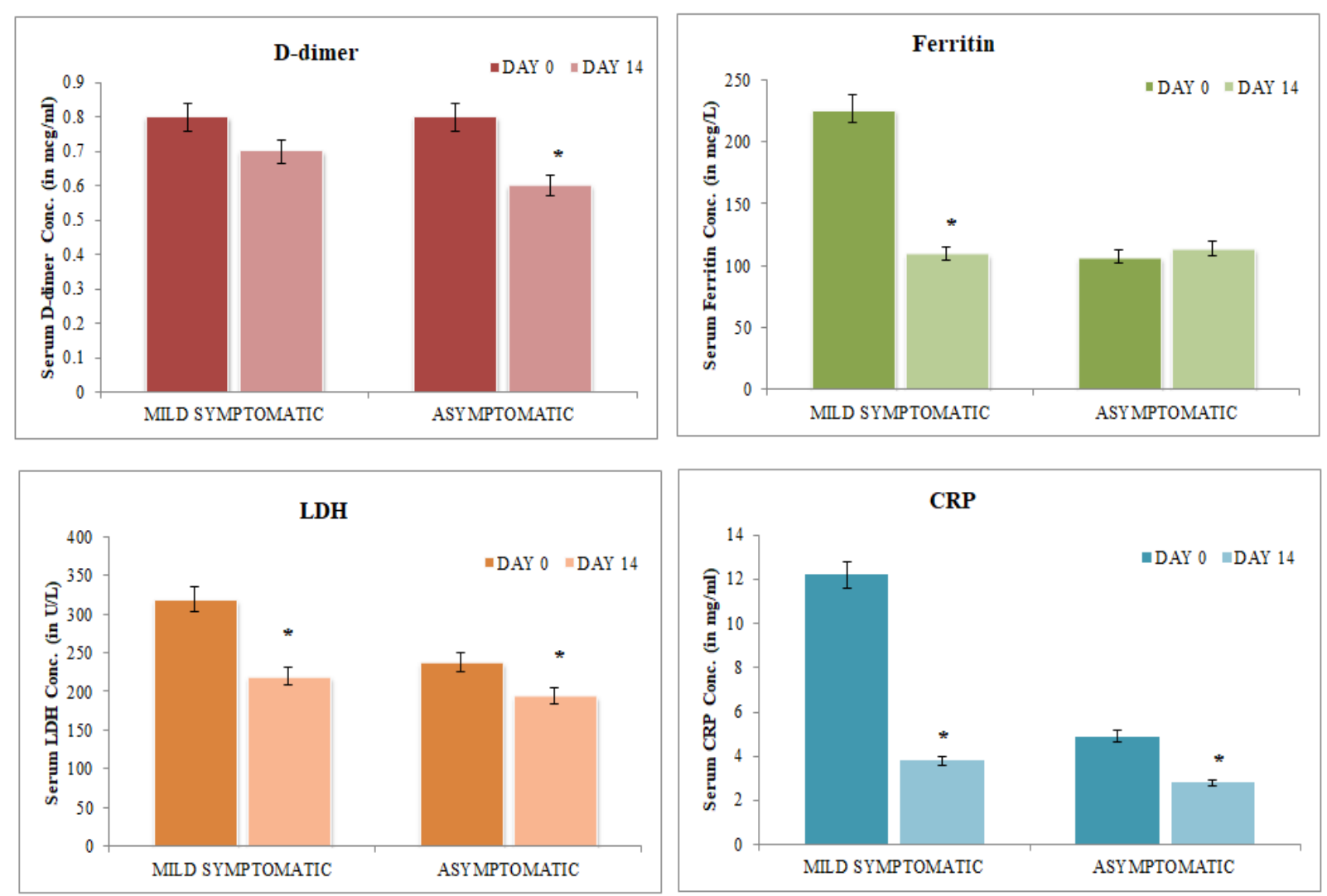

Figure 3: D-dimer, ferritin, LDH and CRP values were compared for mild symptomatic and asymptomatic arms on day 0 and day 14. 
We found that lack of control arm receiving standard of care treatment alone is the limitations for this study. A randomized, double blind, placebo controlled with standard of care treatment arm would have given more insights about the trial.

\section{CONCLUSION}

Early recovery from signs and symptoms was observed in most of the patients. Sri Sri Tattva ${ }^{\mathrm{TM}}$ immunity products has also demonstrated an excellent improvement and immune markers including TNF-alfa, IL-6, IFN-beta, Ddimer, ferritin, LDH and CRP in COVID-19 patients. The final results of this study showed that Sri Sri Tattva ${ }^{\mathrm{TM}}$ immunity products reduced the time to clinical cure and number of days in achieving negative RT-PCR by early viral clearance when given along with standard of care thus reducing the duration of hospitalization. In the present study $82 \%$ of COVID-19 patients cleared the virus within 10-14 days of study period. None of the patients progressed to severe COVID 19 and none of the patients succumbed to the disease. This clearly indicates that Sri Sri Tattva ${ }^{\mathrm{TM}}$ immunity products when administered orally along with standard of care has definitive role in the management of COVID-19 patients.

\section{ACKNOWLEDGEMENTS}

We would like to acknowledge Mr. Arvind Varchaswi, Managing Director and Mr. Tej Katpitia, CEO, Sriveda Sattva Private Limited (Sri Sri Tattva), India for their support in conducting the study. We also thank the investigator's team who supported in the complete documentation and also in the regular patient follow-ups. We would also like to extend our thanks to Dr. Jayanthi C $\mathrm{R}$, Director cum Dean, BMC and all the BMC staff and hospital administration for providing us with necessary facilities and permissions for recruitment of trial subjects.

\section{Funding: No funding sources}

Conflict of interest: None declared

Ethical approval: The study was approved by the Institutional Ethics Committee

\section{REFERENCES}

1. Astin JA. Why patients use alternative medicine: results of a national study. JAMA. 1998;279(19):1548-53.

2. Tabish SA. Complementary and alternative healthcare: is it evidence-based? Int J Health Sci. 2008;2(1):21475465.

3. Mike S. Complementary and alternative medicine. Encyclopæd Britannica. 2018;3:1-48.

4. Gaudreau A, Hill E, Balfour HH, Erice A, Boivin G. Phenotypic and genotypic characterization of acyclovir-resistant herpes simplex viruses from immunocompromised patients. $\mathrm{J}$ Infect Dis. 1998;178(2):297-303.

5. Safrin S, Cherrington J, Jaffe HS. Cidofovir: review of current and potential clinical uses. Advan Experiment Med Biol. 1999;458:111-20.

6. Rajesh A, Chawla R, Marwah R, Arora P, Sharma RK, Vinod K. Potential of complementary and alternative medicine in preventive management of novel H1N1 flu (swine flu). Pandemic: thwarting potential disasters in the bud. Evid Based Complement Alternat Med. 2011;2011:586506.

7. Liang-Tzung L, Wen-Chan $\mathrm{H}$, Chun-Ching L. Antiviral natural products and herbal medicines. J Tradition Complement Med. 2014;4(1):24-35.

8. Akshada AK, Rajendra CD, Chandrakant SM. Natural products in drug discovery. Pharmacog Med Plants. 2019.

9. Xiao AT, Tong YX, Gao C, Zhu L, Zhang YJ, Zhang S. Dynamic profile of RT-PCR findings from 301 COVID-19 patients in Wuhan, China: a descriptive study. J Clin Virol. 2020;127:104346.

10. Xiaoqiang C, Longfei H, Yan Z, Weiyu H, Zhou L, Aiwu K. Specific ACE2 expression in cholangiocytes may cause liver damage after 2019-ncov infection. Biorxiv. 2020;2:931766

11. Li W, Moore MJ, Vasilieva N, Sui J, Wong SK, Berne $\mathrm{MA}$, et al. Angiotensin-converting enzyme 2 is a functional receptor for the SARS coronavirus. Nature. 2003;426:450-4.

12. Lei F, Liu Y, Zhou F, Quan J, Zhang P, Zhu L, et al. Longitudinal association between markers of liver injury and mortality in COVID-19 in China. Hepatol. 2020;72(2):389-98.

13. Brandon MH, Gaurav A, Johnny W, Stefanie B, Jens $\mathrm{V}$, Mario P, et al. Lactate dehydrogenase levels predict coronavirus disease 2019 (COVID-19) severity and mortality: a pooled analysis. Ame J Emerg Med. 2020;38(9):1722-6.

14. Shi J, Li Y, Zhou X, Zhang Q, Ye X, Wu Z, et al. Lactate dehydrogenase and susceptibility to deterioration of mild COVID-19 patients: a multicenter nested case-control study. BMC Med. 2020;18(168):74.

15. Wenjie T, Xiang Z, Xuejun M, Wenling W, Peihua N, Wenbo $X$, et al. A novel coronavirus genome identified in a cluster of pneumonia cases-Wuhan, China 2019-2020. China CDC Weekly. 2020;2(4):61-2.

16. Chaolin H, Yeming W, Xingwang L, Lili R, Jianping $\mathrm{Z}$, Yi H. Clinical features of patients infected with 2019 novel coronavirus in Wuhan, China. The Lancet. 2020;395:497-506.

Cite this article as: Reddy MRK, Venkatesh KRH, Jothilakshmi J. Herbal immunomodulators as add on treatment in asymptomatic and mildly symptomatic COVID-19 confirmed cases: findings from a prospective single centre clinical trial. Int J Basic Clin Pharmacol 2021;10:653-9. 